\title{
Ein Meisterliches Künstlerporträt: Frans Hals' Ostade-Bildnis
}

Es gibt aus dem siebzehnten Jahrhundert etliche Beispiele, daß Künstler ihre Zunftgenossen, Meister, Schüler und Kollegen gemalt haben-am berühmtesten ist das van Dycks; und Houbrakens Erzählung, daß sich Hals und van Dyck gegenseitig gekonterfeit hätten, spricht zumindest für die Beliebtheit der Gattung 'Künstlerporträt'. Wir wissen von Hals, daß er mehrere andere, meist jüngere Künstler gemalt hat, so Jan Miense Molenaer, Judith Leyster, Frans Post, Vincent Laurensz. van der Vinne und Leendert van der Cooghen. Von einem Porträt seines erfolgreicheren Schülers und Haarlemer Mitbürgers Adriaen van Ostade gibt es dagegen keine urkundliche Erwähnung. Doch eine glaubhafte Tradition hat Ostades Bildnis im Schabkunstblatt von J. Gole überliefert, das auf eine Vorlage von Cornelis Dusart zurückgeht ${ }^{1}$. Dieses - auch in Dusarts Nachlaß erwähnte ${ }^{2}$-Bild des erst 1660 geborene Schülers zeigt den 1685 verstorbenen Meister mit einem für einen 75-jährigen ungewöhnlich jugendlichen Aussehen. Eduard Trautscholdt ${ }^{3}$ folgert konsequent daraus: 'Eine unverkennbare Ähnlichkeit Adriaens auf diesem Schabkunstblatt mit seinem urkundlich belegten Selbstbildnis rechts auf dem Vierfigurenbild des Museums Bredius 'De familie De Goyer met de schilder', das um oder etwas nach 1650 angesetzt wird, läßt vermuten, daß Dusarts Gemälde auf ein Bildnis zurückgeht, das Adriaen im Alter von 40-45 Jahren zeigte'. Trautscholdt vermutete als Vorlage ein Terborch-Porträt (H.d.G.285, Gudl.136), das I. Q. van Regteren Altena als Ostade darstellend angesprochen hatte und schloß, 'daß der Schüler auf ein Porträt seines Lehrers (oder auf mehrere Bildnisaufnahmen) aus den 50-er Jahren des Jahrhunderts zurückgriff, um ihn nachschaffend in seinen besten Jahren darzustellen. Dabei mag die auf dem Stich auffällig stattliche Lockenperïcke mehr der Mode um 1700 angepaßt sein, als es der oder den Vorlagen entsprach, sei es nun, daß dies auf Dusart oder Gole zurückzuführen wäre.'

Die Identifizierung des Terborch-Modells mit Ostade scheint uns nicht haltbar, da zwei sehr verschiedene Physiognomien dargestellt sind: die Höhe der Backenknochen, Wölbung und Proportion von Nase und Stirn, Formung der Nasenflügel (die bei Ostade typischerweise seitlich hochgeschoben erscheinen), geben die wichtigsten Unterscheidungsmerkmale. Wie sehr Trautscholdt aber recht gehabt hat mit seinen ganz allgemeinen Überlegungen, zeigt der Vergleich zwischen dem Stich und dem unmittelbar diesem zugrundeliegenden Gemälde Hals’. Die in den Details nahezu vollständige Überein-

1. Über dieses und ein anderes Schabkunstblatt, das Gole nach einer Selbstporträtvorlage Ostades geschaffen hat, siehe: E. Scheyer, Portraits of the Brothers van Ostade, The Art Quarterly II, 1939, S. 134-141 und Eduard Trautscholdt, Zu einer Bildniszeichnung des
Adriaen van Ostade, Miscellanea I. Q. van Regteren Altena, Amsterdam 1969, S. 152-154.

2. Bredius, Künstler-Inventare, Bd. I. Den Haag 1915, S. 45, Nr. 11.

3. Trautscholdt, a.a.O., S. 153. 
stimmung macht Einzelhinweise unnötig. Der Stich stellt einen—durch Seitenverkehrung beim Drucknach links gewendeten selbstbewußten jungen Mann dar, geschmückt mit 'stattlicher Perücke, deren Locken bis über die Schultern fallen. Der weite, vorn offene Mantel läßt ein geschlungenes seidenes Halstuch sehen. Das Porträt erweckt den Eindruck einer gewissen modischen Eleganz'4 ${ }^{\prime}$. Der weich im Sinne der 'Negligé-Mode' fallende Überwurf verstärkt den Anschein des Bewußten und Selbstsicheren aus dem Kontrast zwischen pompöser Nachlässigkeit des Körperlichen und der auf den Betrachter gerichteten Aufmerksamkeit des Gesichts.

Ganz wesensverschieden erscheint van Ostade auf dem ursprünglichen Porträt Hals'. Dieses 94 x $75 \mathrm{~cm}$ messende hochformatige Halbfigurbild läßt sich seinen stilistischen Merkmalen nach 1643-44 datieren. Es zeigt den entsprechend etwa 34-jährigen jungen Maler in nachdenklicher Haltung vor dem Beschauer verweilend. Er steht, die rechte Hand halb geöffnet und seitlich an den Körper gelegt, die linke - behandschuht und einen Handschuh locker haltend - entspannt herunterhängend. Vor grauem Hintergrund hebt sich die dunkle Körpersilhouette ab. Die Außenkonturen sind nicht kantig umrissen, sondern zerbröckeln in unregelmäßiges Zickzack oder sind als erkennbare einzelne Pinselzüge von der Flächenfigur abgehoben. Ebenso sind die Lichthöhungen und Schattenkanten der Mantelfalten als flache Pinselstreifen ausgeführt: auf einem mittleren Grauton liegt lichteres Grau oder Schwarz. Im Gegensatz zu dieser betont flächigen Lösung der Gewandpartien bleibt im Gesicht eine weich abgestufte Licht- und Schattenmodellierung erhalten als Unterlegung der bewußten-als Pinselzüge kenntlichen-Akzentuierungen. Auf einige charakteristische Hauptlinien an Augen, Mund und Nase verteilen sich die wenigen sehr dunklen oder sehr hellen Hervorhebungen. Jedoch fallen diese nicht zusammen mit dem Gesamtverlauf der 'objektiven' Begrenzungen: der Augen- und Lidränder, der Zeichnung von Nasenflügeln und Mundlinie. Den Lichteinfallswinkel als Motivation benützend sind sie jeweils in eine Richtung wegverschoben, in der die Spannungslinien der sich bewegenden Gesichtsmuskulatur hervortreten. Zugleich sind diese schmalen Streifen so gewählt, daß der Pinselauftrag einen im Flächenbild parallelen, quasi rhythmisch sich wiederholenden Verlauf erhält. Das gleichzeitige Betonen der stets mit einer Kante oder Ecke 'herausspringenden' Konturen dieser vorrangig placierten Akzente erweist das Bewußtwerden des Beobachtens als eines momentbedingten Vorgangs. Einerseits wird so höchst veristisch die verhaltene Spannung und Bewegung der Gesichtszüge eingefangen;--sie verbindet sich mit einem träumerisch in die Ferne gerichteten Blick zu einer seltsamen Mischung von freundlicher Skepsis und elegischer Nachdenklichkeit-; andererseits wird der optische Ablesevorgang des Betrachters bewußt, der beim einmaligen Hinsehen nur etwas Vages, Flaues, Flaches entdeckt oder aber etwas ganz Zufallsbedingt-Gestaltloses. Erst die geistige Kombination verschiedener Beobachtungsweisen vermittelt mehr: Wesenszüge des Geschilderten.

In der Rangfolge des geistigen Interesses sind die Hände entsprechend flüchtiger modelliert, aufgebaut aus rhythmisch ineinandergekeilten kurzen Farbstreifen. Nur das Gesicht ist räumlich (!) gesehen, 
mehrfach betrachtet, während der übrigen Erscheinung eine schemenhaft flache, nur ungefähr erfaßte unkörperliche Schattenqualität eignet.

Gerade diese Konzentration aller Bildmittel auf Verdeutlichung des psychischen Ausdrucks hin fehlt einer Werkstattvariante nach dem Washingtoner Ostade-Bild (als Neuerwerbung in der National Gallery of Canada, Ottawa) ${ }^{5}$. Das Verhältnis von Gegenständlichkeit, d.h. Motivkombination und -Rangordnung, mimischem Ausdruck und körperlicher Haltung, und von Art und jeweiliger Graduierung der Sehweise und rhythmischer Organisation der Flächenkomposition bleibt gliederungsarm und ordnungslos. Kopistenhaft sind Motive anderer Bilder anorganisch zusammengefügt, und die Ausführung der Details ist mehr eklektisch übernommene Manier als Stufung und Deutung der zum Thema gewählten Sichtbarkeit. Der Farbauftrag ist-besonders deutlich im Gesicht-von einer zittrigen Unentschlossenheit, einem dünnlinigen Umtasten der Form, das die Gesichtsfläche faserig aufsplittert. Die anatomisch sichere, dezente Vormodellierung des Vorbildes fehlt, das zeigt eklatant die Modellierung von Kinn und linker Kieferseite. Eine große Zahl ungeordneter Detailbeobachtungen der Gesichtsoberfläche ist an die Stelle entschiedener Markierung getreten. Natürlich ist bei diesem, im Formalen so unsicheren Farbauftrag jede ordnende Rhythmik verlorengegangen. Der malerische Schmelz, das in die Andeutung, ins Unausgeführte Zurückweichen nebensächlicher Partien ist durch eine peinlich unräumliche Konturzeichnung ersetzt worden (z.B. die Außenkonturen des Gesichts, das Ohr besonders!). Wo mutige Pinselzüge gesetzt werden, geschieht das an technisch leichteren, unverfänglichen Stellen wie am hellen Kragen. Dabei entstehen 'schwebende Reflexe', sinnlose Couragebeweise dort, wo es nichts herauszuheben gibt. Bei F. Hals dem Älteren sind Akzente dagegen nur dort gesetzt, wo sie als Höhungen eine vorbereitete Steigerung vornehmen können.

Der untere Teil des kleinen Porträtchens ist eine nur wenig veränderte, leicht vereinfachte Nachbildung von Arm, Hand und Stuhl auf dem Bilde des (jüngst identifizierten) Willem Coymans'6 (Washington, National Gallery). Der Vergleich der Hände soll zeigen, wie sichere Auslassung beim Original zur phantasiearmen Strichelei bei der Replik wird. Sogar Stuhl und Ärmelfalten sind in der selben Weise entliehen.

Von dem Ottawa-Bild gibt es eine alte Nachzeichnung von J. G. Waldorp, die bezeichnet ist: FH pinx. J. G. Waldorp del. 1778 und eine weitere ${ }^{7}$ von 1790, bezeichnet: F Hals pinx/J. G. Waldorp del 1790. $\mathrm{Zu}$ der Haarlemer Zeichnung existiert als gezeichnetes Pendant ein Frauenbild, bezeichnet FH pinx 1644 J. G. Waldorp del. 1779. Ob-was zeitlich naheliegt-ein Zusammenhang zwischen diesem Frauenbild und den anderen Porträts bestand, ist nicht mehr zu klären. Zumindest wäre ein weibliches Gegenstück zu einem Ostade-Porträt ziemlich unwahrscheinlich, da Ostades erste Frau 1642 starb und er erst 1657 wieder heiratete. Immerhin gäbe die Datierung des Coymans-Porträts auf 1645 den terminus ante quem non für die in Ottawa befindliche Variante.

6. H.d.G. 168; Valentiner 1923, 225; C. Grimm, Frans Hals, Gesamtmonographie (in Vorbereitung), Nr. 134. Die überzeugende Identifizierung leistete Katrina V. H. Taylor. A Note on the Identity of a
Member of the Coymans Family by Frans Hals, in: Report and Studies in the History of Art, Nation. Gallery Washington 1969, S. $107 \mathrm{ff}$.

7. Siehe dazu auch: Seymour Slive, Katalog der Hals-Ausstellung, Haarlem 1962, Bemerkung zu Kat. Nr. 54. 

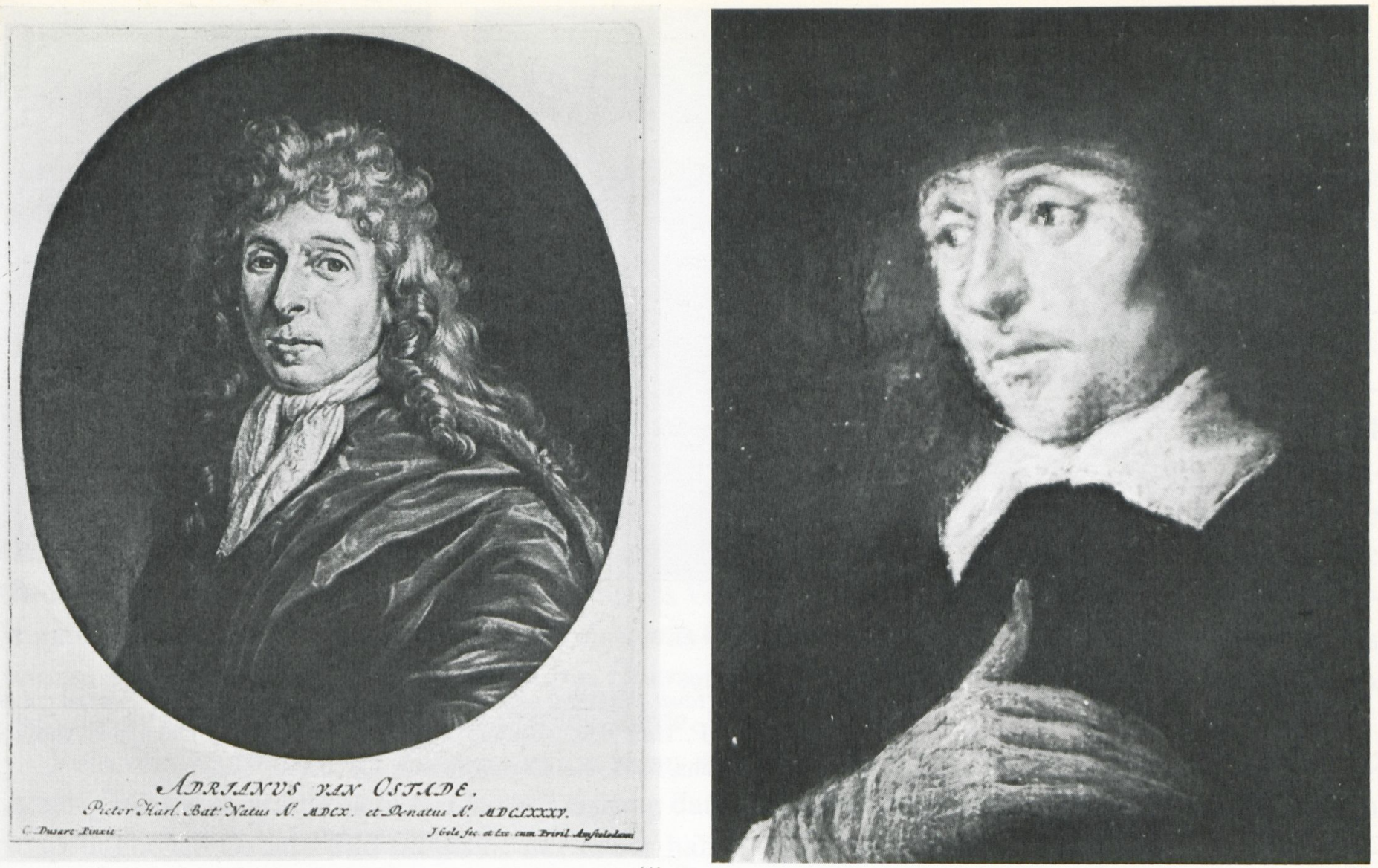

(1)

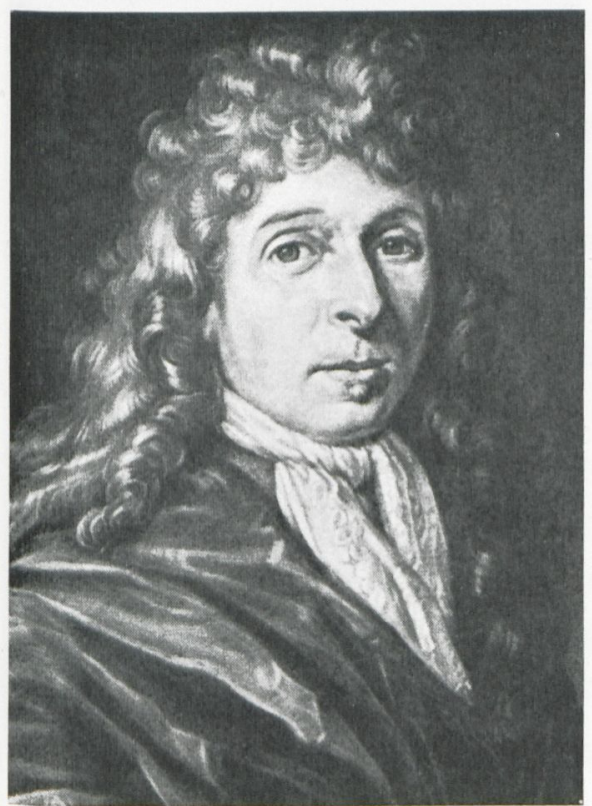

(3)

(1). Bildnis des Adriaen van Ostade. Schabkunstblatt von J. Gole nach Cornelis Dusart. - (2). Selbstbildnis Ostades. Detail aus dem Bild der 'Familie de Goyer mit dem Maler'. Den Haag, Mu-

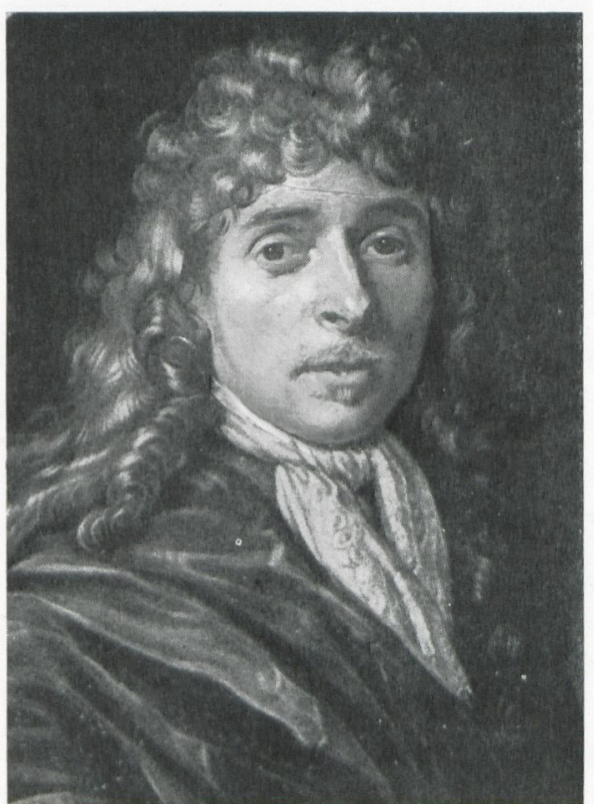

(4)

seum Bredius, um 1650. - (3). Seitenverkehrte Detailvergröszerung aus Abb. 1. -(4). Rekonstruktion des verlorenen Bildes von C. Dusart durch Montage von Gewand und Perücke aus Abb. 1

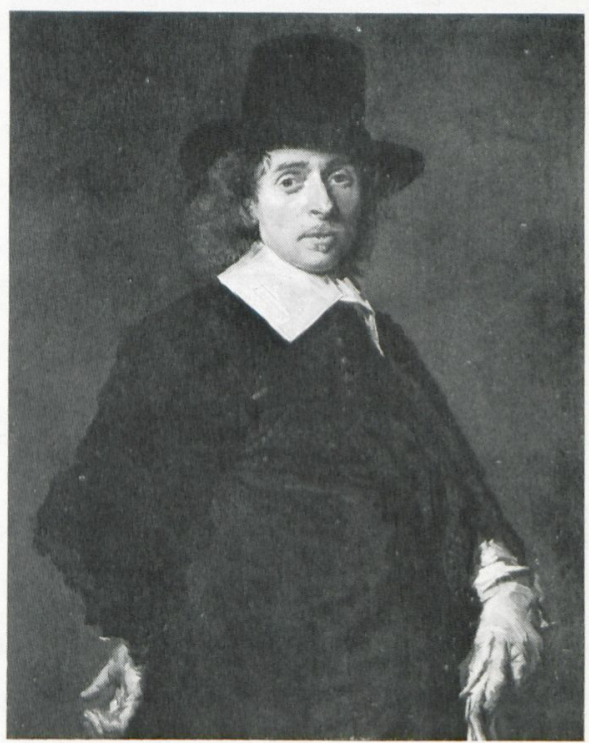

(5)

und Kopf aus Abb. 5.-(5). Frans Hals d. Ä, Porträt des Adriaen van Ostade, 1643/44. Washington, National Gallery of Art. 


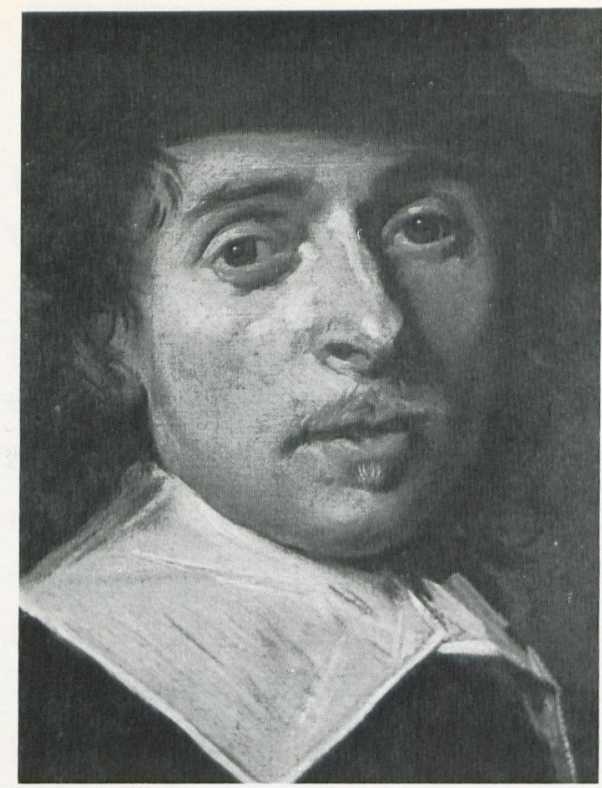

(6)

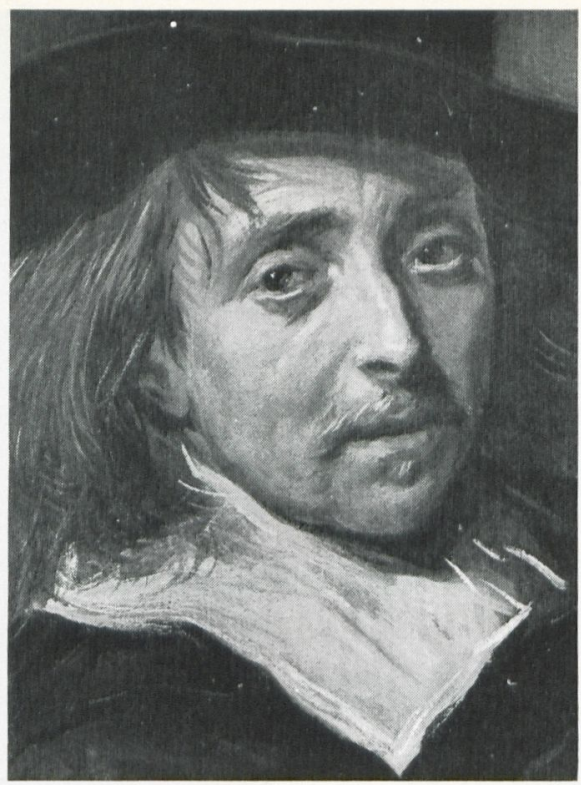

(7)

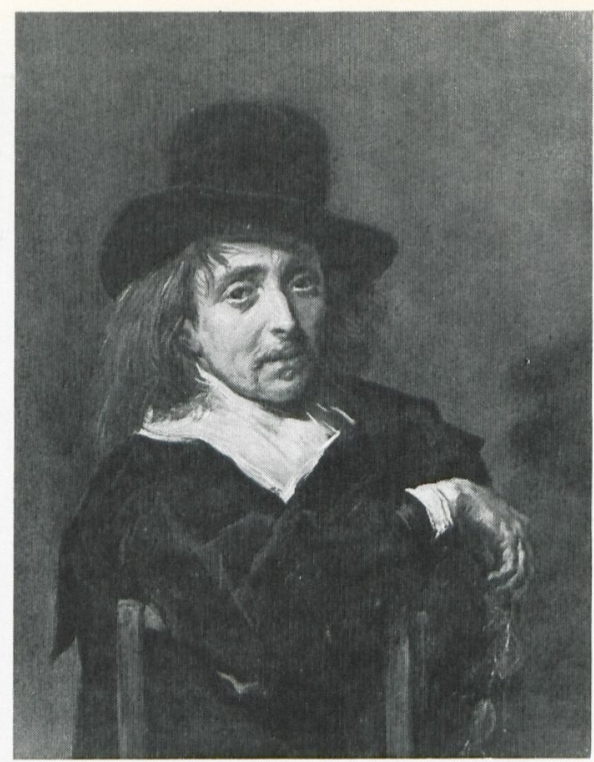

(8)
(6). Detail aus Abbildung 5.-(7). Detail aus Abbildung 8.-(8). Frans Hals der Jüngere (?), Adriaen van Ostade, nicht vor 1645. Ottawa, National gallery of Canada.-(9). F. Hals d. Ä.: Bildnis des Willem Coymans, 1645. Washington,
National Gallery.-(10). Detail aus Abbildung 11.-(11). Detail aus Abbildung 10.

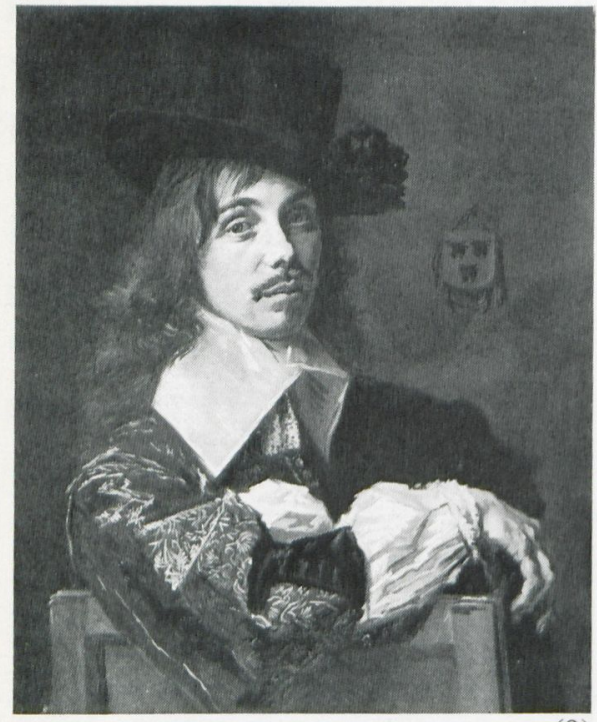

(9)
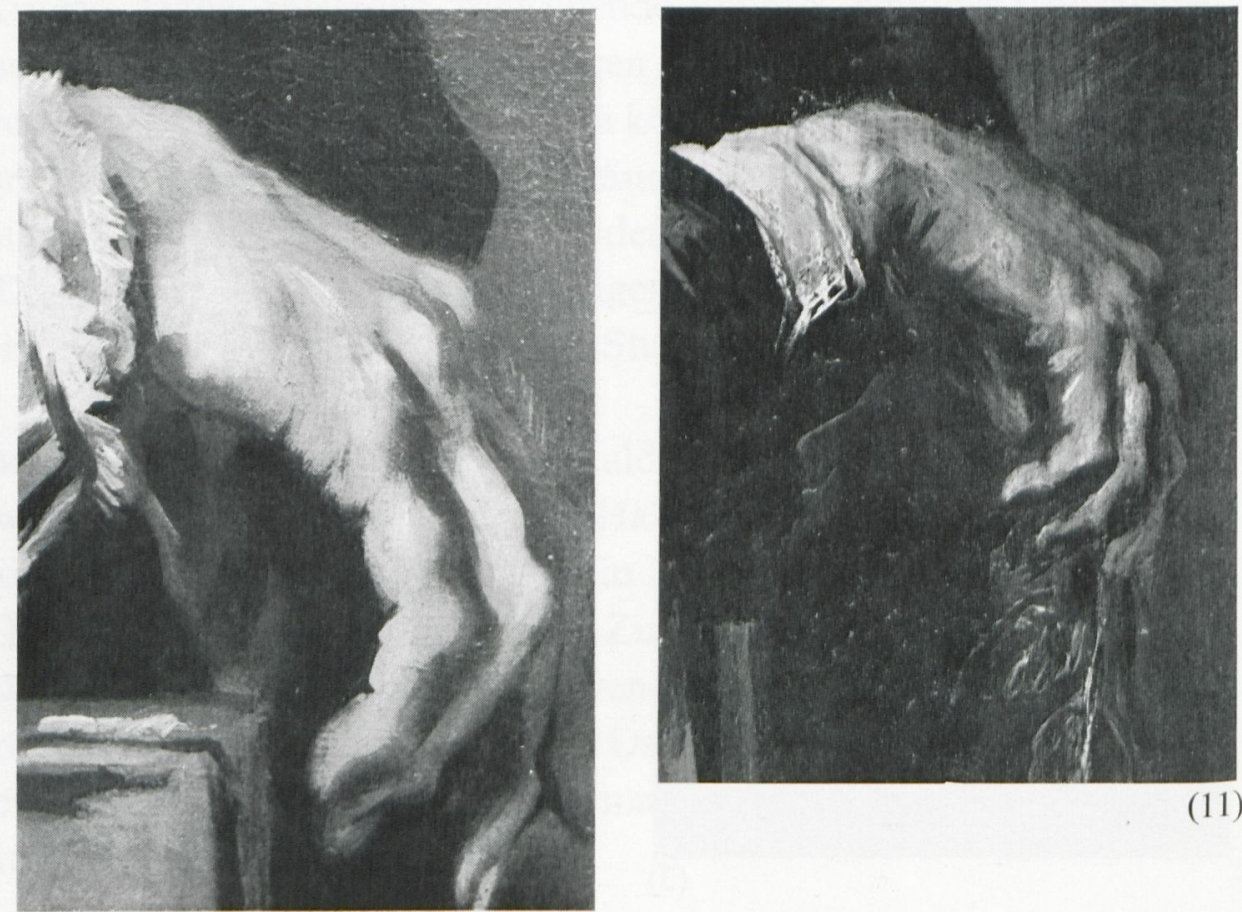

(11)

(10) 
Als Autor der Variante kommt eine Hand aus dem Schülerkreis Hals' in Frage, der wir rund 25 in ähnlicher Manier gemalte Bilder zuweisen zu können glauben ${ }^{8}$. Aus hier nicht weiter ausführbaren Gründen haben wir diese Zahl von bisher Frans Hals d. Ä. attribuierten Werken seinem Sohn Frans Hals dem Jüngeren zugewiesen.

Einige Sachverhalte lassen sich durch die Variantenfolge Hals d.Ä.-Hals d.J.-Dusart erhellen. Wesentlich ist dies die Arbeitsweise und Weiterauswertung bei der Porträtherstellung. In beiden Nachbildungen zeigt sich eine nüchterne Einschätzung des Erinnerungsmediums Porträt. Die sozialrepräsentativen Akzidenzien, Haarmode (auch der Schnurrbart), Kostüm, Haltung werden unbefangen auf den jeweils neuesten Stand gebracht. Die verkleinerte Replik wird einem späteren, von Hals selbst vorgebildeten legeren Haltungstypus angepaßt, während das posthume Schabkunstblatt modisch up to date gebracht wird. Offensichtlich kommt dem Porträt primär die Aufgabe zu, die gesellschaftliche Bedeutung des Individuums zu demonstrieren. Im Verständnis der communis opinio kann im 17. Jahrhundert ein sozialer Würdeanspruch noch als etwas Objektives sichtbar gemacht werden-ganz anders wie im 19. Jahrhundert, wo es nurmehr das Übersetztwerden in die Geltungssphäre des Ästhetischen war, das zu einem teuer erkaufbaren Privileg wurde.

Vom rein reproduktiven Vorgang her gesehen stellt die Kopistenarbeit Dusarts und die Stecherarbeit Goles eine grandios zuverlässige Leistung dar. Dadurch ist uns ein gesicherter Zugang zu dem ursprünglichen Ostade-Bild möglich; nur daher haben wir Kenntnis der Physiognomie dieses bedeutenden Meisters.

Außerordentlich anregend ist die Überlegung, wieweit die uns aus Ostades Werk bekannte künstlerische Wesensart mit dem Eindruck übereinstimmt, den wir aus Hals' Charakterisierung schöpfen. $\mathrm{Daß}$ nicht nur das Physiognomische bei Hals gesehen ist, zeigen die verschiedenen ungleichzeitigen Beobachtungsstufen und die graduelle Betonung auf psychische Bewegungsmerkmale hin. Der uns vorgestellte geistige Typus mit einem distanzbetont-empfindsamen Ausdruck und einer feingliedrig und zartlinig geprägten Physiognomie kontrastiert der Vorstellung vom Bauernmaler Ostade. In gewisser Weise ergibt sich dieser Widerspruch schon von dem Selbstbildnis auf dem Bilde des Bredius-Museums her. Wir haben spätestens von nun an ihn in seiner Inszenierung auch der bäuerlichen Milieuszenen differenzierter zu begreifen. Als einen feinsinnig-abwägenden Meister erweist ihn auch die Neuordnung seines Werks ${ }^{1}$. Zeichnungen und Gemälde verraten einen skeptisch-tiefgründigen Beobachter von Naturvorgängen, die hinter dem Lärmen der Genreszenen ebenso verborgen liegen wie hinter dem Abglanz seiner träumerisch-rembrandtesken Landschaft von 1639 (im Rijksmuseum) 9

Sicherlich läßt sich mit größerer Bestimmtheit sagen, welche Gesichter in den Genreszenen als Selbstdarstellungen gelten können und welche nicht. Denn Idiosynkrasien genügen nicht zur Erklärung von Identitäten, denen offensichtlich ein häufig wiederverwendetes mimisch-physiognomisches Stu-

8. C. Grimm, Hals und die Halsschule, Frans Hals der Jüngere (in Vorbereitung). Auch das von Waldorp gezeichnete Frauenbild wird den ermittelbaren Kriterien nach von uns in diesen Zusammenhang eingeordnet.
9. Bernhard Schnackenburg, Die Zeichnungen der Brüder van Ostade (in Vorbereitung; Neuzusammenstellung und chronologische Werkverzeichnisse). 
dienmaterial zugrundelag. Dabei kann ein Porträttyp aus der Mischung von Beobachtungen an eigenen und an fremden Zügen resultiert haben, ein Ausdruckstyp sein, auf den hin auch das Abbild des eigenen Gesichts verändert worden ist (solchermaßen 'tendiert' das von Gole überlieferte Selbstporträt Adriaen van Ostades in die Richtung eines in den Genrebildern sehr häufigen Typus. Es bietet sich auf diese Weise ein hervorragendes Studienmaterial zum Thema des 'Selbstbildnerischen in der Kunst'10, zugleich ein Beleg dafür, wie wenig diese Idee am Konstitutionstypus ${ }^{11}$ verifizierbar ist.

Individuen sind wohl praeformiert, um eine ganz bestimmte Welterfahrung zu haben und daher andererseits bestimmte Symbolzusammenhänge als verbindlich $\mathrm{zu}$ empfinden, niemals aber, um die von bedeutungslosen, symbolarmen Modalitäten mitbedingten Prägungen der eigenen Gesichtsbildung und Körperstatur in allem und jedem wiederzufinden. Umso mehr haben die Einstellung zur Umwelt und die in Kunstwerken manifeste Deutung der Umwelt miteinander zu tun-hier sei das Bildnis Hals' ein interpretatorischer Wegweiser zum Oeuvre Ostades!

10. G. F. Hartlaub, Das Kunstwerk als Selbstdarstellung des Künstlers, in: G. F. Hartlaub u. F. Weissenfeld, Gestalt und Gestal- tung, Krefeld 1958.

11. Felix Weissenfeld, a.a.O. 